\title{
Frailty research strengthens with biomarker and treatment leads
}

It takes mere seconds for Jonathan Afilalo to predict an elderly patient's risk of developing complications in the days and weeks after heart surgery-and it involves a test that's about as simple as they come. Afilalo, a cardiologist at the Jewish General Hospital in Montreal, asks a patient to walk five meters down the hallway. If that person doesn't make it to the finish line within six seconds, his or her odds of living another six months are lower than patients who can walk faster, Afilalo has discovered. The chances of surgery complications, a second operation or more time on a mechanical ventilator are higher, too (J. Am. Coll. Cardiol. 56, 1668-1676, 2010).

This gait speed test isn't a direct measurement of heart health, though, like other tests administered by cardiologists. Instead, it's a marker of frailty, a concept that has been around in the English language for centuries but has only recently begun to be described more concretely as a medical syndrome. Now, frailty is being integrated into medical tests, researchers are finding causes at the molecular level of the weakening and slowing of the body during aging, and treatments that reverse or prevent frailty could be on the horizon. In addition to surgical outcomes, how frail someone is has been linked to recovery outcomes following pneumonia, stroke, heart attacks and bone fractures, among other maladies.

"Many physicians and surgeons typically thought of frailty as an 'eyeball test", says Afilalo. "But we know now that frailty is actually a biological syndrome that can be objectively measured and used to improve prediction of outcomes."

Different researchers have come up with their own ways of measuring frailty, but one of the most used metrics was developed in 2001 by Linda Fried, now dean of Columbia University's Mailman School of Public Health in New York. Under Fried's frailty scale, patients must meet three of five criteria to classify as frail: unintentional weight loss, exhaustion, weak grip strength, slow walking speed and low physical activity. Other frailty scoring systems consist of longer questionnaires, psychological health measurements or different physical tests.

As frailty has transformed from a descriptive, unscientific word to become a measurable syndrome, researchers have strived to answer two key questions: What health outcomes can frailty scores predict? And how can frailty be treated?

\section{Epic frail} an increasing number of departments are integrating frailty scores into patient assessments, particularly before surgery or chemotherapy treatments. "They're already used to predict a patient's length of stay and organize appropriate social support," says Mayo physiologist Nathan LeBrasseur. But for each new type of surgery or treatment that researchers want to apply frailty scores to, a new study is needed. LeBrasseur, for example, is currently launching a trial to determine the usefulness of frailty scores in predicting the outcomes for elderly patients undergoing surgery to replace or remove part of the colon.

Studies are also ongoing to look at ways to treat frailty. In Australia, rehabilitation doctor Ian Cameron of the University of Sydney recently ran the so-called 'frailty intervention trial', or FIT, an investigation into the effects of exercise programs on reducing frailty measures. The trial enrolled 241 participants aged 71 to 101 who were all classified as frail according to the Fried scale at the outset of the study. After a year, those participants who received ten home exercise sessions with a physiotherapist walked faster and reported higher levels of daily activity than those who received usual care (BMC Med. 10, 120, 2012).

"We found that we could reverse frailty to some extent, and we now think there could be even more benefit treating people who are part of the way down the path to frailty," Cameron says. He and his colleagues are currently planning a trial to test the effects of exercise
At the Mayo Clinic in Rochester, Minnesota,

interventions on those classified as 'prefrail'that is, having some of the criteria of frailty, but not enough that they are considered fully frail yet.

The search is on for drugs that could treat or prevent frailty, too. In mice, LeBrasseur has found that drugs such as rapamycin that increase lifespan-already a widely used endpoint for human clinical trials-generally also increase so-called 'health span', the time period of life before the onset of frailty. But not always, notes Steve Austad, interim director of the Barshop Center for Longevity and Aging Studies at the University of Texas Health Science Center in San Antonio. "Longevity and health and not necessarily intricately linked," he says.

Some researchers would now like to see frailty more closely scrutinized in animal models (see page 518); others want frailty recognized by drug regulators as an endpoint in future human clinical trials involving experimental agents designed to delay the effects of aging. However, reliably and systematically gauging frailty in patients could be tough. A 2007 working group convened by the NIA came to the conclusion that many endpoints used to determine functional declines associated with aging - not only broad frailty scores, but stair-climbing tests and various other walk tests-have not been studied enough for researchers to have a sense of what level of improvement on these tests constitutes a positive result (J. Gerontol. A Biol. Sci. Med. Sci. 63, 160-164, 2008). With such a weak evidence base, continued research on frailty should help strengthen the field.

Sarah CP Williams

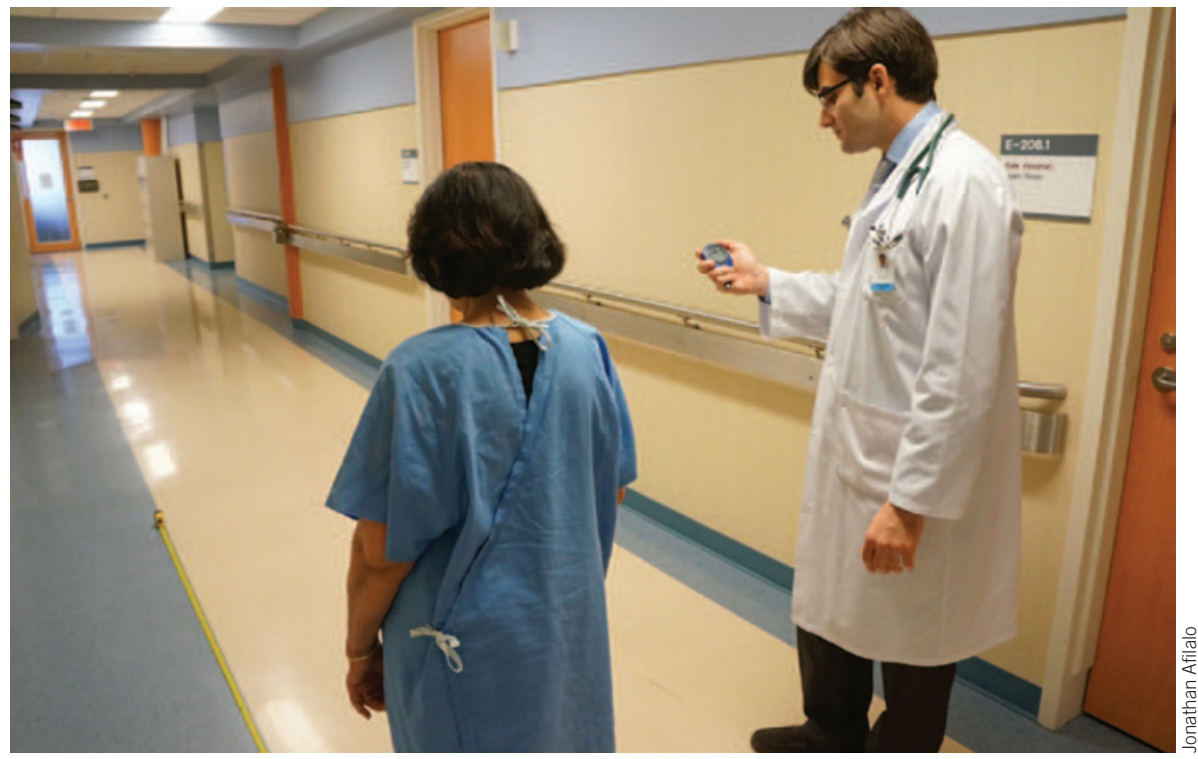

A step in the right direction: Jonathan Afilalo (right) clocks a patient's performance on the gait speed test. 